\title{
Cytochrome P450 Gene in Silkworm (Bombyx mori): A Review Article
}

\author{
Endale Hailu ${ }^{1,2,3}$, Yuehua Zhang ${ }^{1,2^{*}}$ and Guodong Zhao ${ }^{1,2}$ \\ ${ }^{1}$ Jiangsu University of Science and Technology, China \\ ${ }^{2}$ Sericultural Research Institute, Chinese Academy of Agricultural Sciences, China \\ ${ }^{3}$ ICIPE Ethiopia YESH Project Field Assistant Arba Minch Gamo Gofa Zone, Ethiopia
}

*Corresponding author: Yuehua Zhang, Jiangsu University of Science and Technology, Zhenjiang Jiangsu 212003, China.

Received Date: September 06, 2018

Published Date: September 28, 2018

\begin{abstract}
To understand features of cytochrome P450 genes in silkworm, the advances in silkworm genome projects created a new genome wide analysis tool that enables us to seek cytochrome P450 genes constitutes and their diversities. This has provided not only an opportunity for divergence in their substrate specificity identification, but also for divergence in their regulatory attributes, such as differing patterns of induction, tissue specific expression, and developmental expression. However, different researchers so far made different efforts to identify number of cytochrome p450 genes in Bomboxy mori, they came up with slightly different numbers which ranges from 79 to 86 putative cytochrome $\mathrm{p} 450$ genes. Then, it urges us to undertake further investigate in order to arrive at certain consensuses putative cytochrome 450 genes number.
\end{abstract}

\section{Introduction}

Cytochrome P450 monooxygenases (termed CYPs or P450s originated from spectrophotometric peak at wavelength of the absorption maximum of the enzyme at $450 \mathrm{~nm}$ ) are collective name for the heme-protein containing enzymes that constitute a ubiquitous of hydrophobic, cysteinato-heme enzymes. They are one of the oldest and largest super-family. For their enzymatic reaction a variety of small and large molecular substrates are utilized. In general, terminal oxidase enzymes in electron transfer chains, categorized as P450-containing systems [1-4].

As the means of oxidation, P450 uses molecular oxygen, inserts one of its oxygen atoms into a substrate, and reduces second oxygen to a water molecule, using two electrons that are provided by NAD(P)H via a reductase protein. Since only one of the two oxygen atoms, initially present in $\mathrm{O}_{2}$, remains in oxidized substrate which result in P450s as monooxygenases. Molecular oxygen, itself, is unreactive toward organic molecules at low temperatures either due to spin forbiddingness or to high barriers. Consequently, living systems mainly use enzymes that modify dioxygen to form capable of performing desired oxidation reaction. This modification can be achieved by metal-dependent oxygenases, like cytochromes P450 or non-heme metalloenzymes (e.g., methane monooxygenase), or by flavin-containing enzymes that do not possess a metal-based prosthetic group [4-7].
Indeed, P450s are important metabolic enzymes involved in metabolism not only of a wide range of endogenous compounds such as fatty acids, steroids, hormones or vitamins, but also exogenous substrates such as drugs, chemicals including environmental pollutants, such carcinogens as polycyclic aromatic hydrocarbons, and pesticides as well as plant toxics. Consequently, they are found virtually in all aerobic organisms, including organisms as diverse as in insects, plants, mammals, birds and bacteria [1,5-9]. Recently, due to the advancement in genome sequencing technology a huge number of P450 enzymes (more than 21,000 sequences of P450 genes) are accessible in the P450 database from all kingdoms of life [1]. The diversify of sequences encoded by a multiplicity genes, which have been arisen by gene duplication and adaptive diversification results in functional versatility of the p450 superfamily i.e., gene duplication is basically a corner stone for evolution of gene family [3].

The crucial roles of $\mathrm{P} 450 \mathrm{~s}$, in insects are synthesis and degradation of insect hormones including ecdysteroids and juvenoids and the detoxification or activation of such chemicals as plant toxins and insecticides in turn that is leading to increased resistance in insect populations. So far, most of them were isolated from dipteran and lepidopteran insect species and more specifically from pest or diseases vector species. So in insects, P450 
genes can be assigned to one of four clades: CYP2, CYP3, CYP4 and the mitochondrial CYP clade. The CYP3 clande is subdivided again into the CYP6, CYP9, CYP28, CYP308-310, CYP317, CYP321, CYP324, CYP329, CYP332, CYP336-338 and CYP345-348 families. Indeed, Many CYP9 family members are known to participate in detoxification pathways associated with insecticide resistance. Hence, they are considered as key enzymes in order to visualize many metabolic pathways and based on these signals that enable to formulate desirable remedial actions[1, 10-11]. Having these in mind, this review aimed at P450s in silkworm species.

\section{Cytochrome P450 Genes in Bombyx Mori}

Based on the advances in silkworm genome projects, a new genome wide analysis of cytochrome P450s genes was performed by different scientists. Consequently, their finds revealed that Bombyx mori genome has not similar putative $\mathrm{p} 450$ gene numbers. That is $\mathrm{Li}$ and his colleagues were discovered that 86 putative P450s in silkworm genome, which are thought to belong to 32 subfamilies. Further, they mad a comparative genomic analysis with Drosophila melanogaster, then the result revealed that the two insects have some similar P450 distribution patterns but still have some obvious differences. Especially, the diverse distribution exists in 7 p450 subfamilies, which are CYP4A, CYP4C, CYP4D, CYP6A, CYP6AE, CYP6B and CYP9A [11]. But, Li and his colleagues in other study, they came up with different result of predication number of putative p450s which was predicted that the genome of silkworm, Bombyx mori, has at least 79 P450 genes; however, P450 genes that are related to the catabolism of exogenous compounds were not reported [12]. On the contrary, Ai and his colleagues predicted that based on the same genome project and analysis of cytochrome P450 genes they obtained a total of 84 CYP-related sequences which could be classified into 26 families and 47 subfamilies according to standard nomenclature. According to their report out of total eighty four genes, seventy eight of them were appeared to be functional and six were probable pseudogenes. Furthermore, their result revealed that the distribution of Bombyx mori P450s in the genome depicted that most of them are tandem arranged on chromosomes, while merely 34 genes were present as singletons, with 8 clusters including 3 or more than 3 genes. On the other hand, they came up with B. mori and H. armigera CYP9A genes clustered in species-specific groups of three P450s, with the exception of the orthologous CYP9A22/CYP9A14 pair, which indicates that the gene expansions are independent species-specific events relative to the last common ancestor. As different phytophagous insects, $B$. mori and $H$. armigera encounter many predictably different chemistries and xenobiotics in their respective host plants and their distinct environments [3]. Hence, due to these dynamic and selective pressures on gene copies mechanisms exposed them to engage into a gradual modification which will create genes with specific functions than their precursors. In general, we infer that these gene evolution scenario will drive insect P450s to diversify rapidly.

\section{Conclusion}

Even though different studies were resulted in different number of p450 genes, the attempt made in identification of putative cytochrome p450 gene numbers and their expression profiles provides new insights into the similarities and differences in the structure, evolution, expression, and functions of genes, and raises many interesting questions which will need further scrutiny and the orthologous relationships among different insect CYP9A genes as well. Hence, it needs further investigation at least to arrive on specific number of cytochrome p450 genes in Bombyx mori to avoid ambiguities.

\section{References}

1. Moustapha SI (2014) Molecular characterization and functional analysis of Cytochrome p450 genes in the yellow fever mosquito aedes aegypti (diptera: Culicidae). K-State Electronic Theses, Dissertations, and Reports pp. 1-166.

2. https://en.wikipedia.org/wiki/Cytochrome_P450

3. Ai J, Yu Q, Cheng T, Dai F, Zhang X, et al. (2009) Characterization of multiple CYP9A genes in the silkworm, Bombyx mori. Mol Biol Rep 37(3): 1657-1664.

4. Shin J., Kim J-E, Lee Y-W and Son H (2018) Fungal Cytochrome P450s and P450 Complement (CYPome) of Fusararium graminearum. Journal of Toxins 112; doi:10.330/toxins10030112: 1-19. (MDPI)

5. Meunier B, IP de Visser S, Shaik S (2005) Mechanism of Oxidation Reactions Catalyzed by Cytochrome P450 Enzymes. American Chemical Society 104(9): 3947-3980.

6. Bhagawati N. and Mahanta R. (2016) Changes in Cytochrome P450 Activity in 4th and 5th instar Larva of Samia ricini on Exposure to the two Organo phosphorous Pesticides, Malathion and Dimethoate-A Comparative Study. IJARSET 3(12) ISSN: 2350-0328: 2987-2996.

7. Notonier S, Meyers A, Jayakody LN (2016) An Overview of P450 Enzymes:Opportunities and Challenges in Industrial Applications. Enz Eng 5: 152.

8. Williams NS, Dunham E, Bradfield AC (2005) Induction of Cytochrome P450 Enzymes. Plenum Publishers 324-346.

9. Jeffrey GS (2008) Insect cytochrome P450s: Thinking beyond detoxification. Department of Entomology, Comstock Hall, Cornell University, Ithaca New York 14853-0901, USA, pp. 117-124

10. Li B, Xia Q, Lu C (2005) Analysis of cytochrome P450 genes in silkworm genome (Bombyx mori). Sci China 48(4): 414-418.

11. Wang LR, Liu WS, Baerson RS, Qin Z, Ma Z-H, et al. (2018) Identification and Functional Analysis of a Novel Cytochrome P450 Gene CYP9A105 Associated with Pyrethroid Detoxification in Spodoptera exigua HübnerInt. J Mol Sci 19(3): 1-13.

12. Li B, Zhang H, Ni M, Wang BB, Li FC, et al. (2014) Identification and characterization of six cytochrome $\mathrm{P} 450$ genes belonging to CYP4 and CYP6 gene families in the silkworm, Bombyx mori. Molecular Biology Report 41(8): 5135-5146. 